\title{
Von der ,epileptischen Kapriole‘ zum Nobelpreis: Die Blechtrommel als Paradigma der deutschsprachigen Literaturkritik
}

\author{
Veronika Schuchter
}

\begin{abstract}
The reception of Günter Grass's novel The Tin Drum in Germany has been both controversial and dynamic. The analysis of newspaper articles from five decades shows that the process of canonisation is divided in four phases: The initial reception in Germany after the first publishing in 1959 is characterized by controversial debates, moving along moral, not aesthetic lines. The first re-reading of the novel is initiated by Volker Schlöndorf's screen adaptation in 1979. It shows the advanced stage of canonisation of the text whose literary qualities are no longer questioned. The award of the Nobel Prize in Literature in 1999 is recognized as success exclusively of The Tin Drum, while Grass's other literary achievements are diminished. In this third phase of reception the most distinctive characteristic of the reception in Germany becomes apparent: the diametrically opposed development of the author and his work. While The Tin Drum is considered untouchable, it's author has lost symbolic capital, mostly because of his political activism. In the last phase, on the occasion of the novel's fiftieth anniversary, The Tin Drum is celebrated as the most important German book in the second half of the 2oth century. The accompanying articles are characterized by an almost sentimental tone, showing that the view of literary criticism increasingly narrowed to a few aspects, an almost schematic form of interpretation, repeating the same stereotypes over and over again.
\end{abstract}

Ob Kritiker und Romanciers weltweit die Frage nach dem „bedeutendste[n] Roman der Weltliteratur in der zweiten Hälfte des 20. Jahrhunderts “1 tatsächlich mit Die Blechtrommel beantworten würden, wie Volker Neuhaus etwas eurozentristisch vermutet, lässt sich leider nicht nachprüfen. Betrachtet man den internationalen Siegeszug, den Günter Grass' Debütroman gleich nach seinem Erscheinen 1959 antrat, ist es zumindest nicht völlig auszuschließen.

$1 \quad$ Neuhaus (2010) 5 .

(C) VERONIKA SCHUCHTER, 2016 | DOI 10.1163/9789004291898_004

This is an open access chapter distributed under the terms of the CC-BY-NC License. 


\section{Günter Grass - Der Blechtrommler}

Die Rezeptionshistorie der Blechtrommel liest sich wie ein Abbild der Karriere ihres Autors, schwankend zwischen Skandalisierung auf der einen und Geniekult auf der anderen Seite. Grass polarisiert mit seinen literarischen Texten, seinem politischen Engagement, seinen autobiografischen Geständnissen. Skandale prägen seine gesamte Karriere seit seinem ersten Roman, der ein Stachel im Fleisch der konservativ geprägten deutschen Nachkriegsgesellschaft unter Adenauer war. Von den einen wurde es als blasphemisches und pornographisches Werk verteufelt, von den anderen als Neubeginn einer selbstbewussten deutschen Literatur gefeiert, die literarisches Engagement mit ästhetischer Raffinesse zu vereinen weiß: „Für die einen

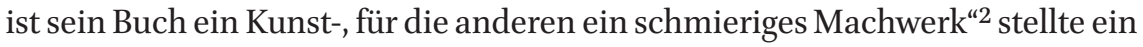
Rezensent schon damals fest. Wenige Karrieren waren so wechselhaft wie jene des 1927 in Danzig geborenen Schriftstellers: Preise wurden ihm zunächst zu-, dann aus politischen Gründen wieder aberkannt, die Blechtrommel zunächst verrissen, dann hochgelobt, bei Das weite Feld gestaltete sich der Prozess genau umgekehrt, nachdem Kritiker-Papst Marcel Reich-Ranicki das Werk auf dem Spiegel-Cover symbolträchtig in der Luft zerfetzte.

Jahrzehntelang als Paradeintellektueller das personifizierte schlechte Gewissen der BRD, hartnäckig die Konfrontation mit der Vergangenheit als Tätervolk einfordernd, wurde Grass' Integrität nach seinem beiläufig vorgebrachten Geständnis in seiner Autobiografie Beim Häuten der Zwiebel, als 17-Jähriger Mitglied in der Waffen-ss gewesen zu sein, selbst fragwürdig. Gegen den einstigen Trommler für die Konfrontation mit der nationalsozialistischen Vergangenheit auf Ebene des ,kleinen Volkes', des Spießbürgertums, wurden Antisemitismusvorwürfe laut. Zuletzt wurde im Kontext der Debatte um das äußerst umstrittene Gedicht, Was gesagt werden muss', zeitgleich veröffentlicht am 04. April 2012 in der Süddeutschen Zeitung, El País und La Repubblica, auf die Blechtrommel referiert. „Von allen guten Geistern verlassen“3 ${ }^{\text {kommen- }}$ tierte die Neue Zürcher Zeitung, „Es ist ein ekelhaftes Gedicht“4 ließ Marcel Reich-Ranicki in der $F A Z$ verlauten, in Israel wurde er zur Persona non grata erklärt. Auch in der hitzigen Debatte zeigte sich wieder: Wenn es um Grass geht, geht es um die Blechtrommel. Grass' ss-Mitgliedschaft und seine Ausfälle gegen Israel - die Antisemitismusvorwürfe entbehren indes auf literarischer Ebene jeder Grundlage - lassen sich nur schwer integrieren in das über

2 Gerhard Schüler, zitiert nach Görtz (1984) 126.

3 Bucheli (2012).

4 Weidermann (2012). 
Jahrzehnte verfestigte Bild des Schöpfers des wichtigsten antifaschistischen Nachkriegsroman Deutschlands.

Die Blechtrommel und ihr Autor sind nicht voneinander zu trennen, dennoch haben die beiden eine diametrale Entwicklung durchgemacht: Während Günter Grass nach dem Höhepunkt des Literaturnobelpreises beständig symbolisches Kapital einbüßte, ist sein Debütroman unantastbar geworden. Sein größtes Werk ist dem Autor über den Kopf gewachsen. Rolf Michaelis hält fest:

Die kritische Meinung über Grass - und sein Werk - wird oft weniger in Form literarischer Kritik geäußert als in Glossen, Kommentaren, giftigen, hämischen Fußnoten zu Auftritten von Grass. Damit muß leben, wer sich so vehement ins öffentliche Leben mischt. Nur: Im Fall Grass beschädigt die bös herablassende Art der Kommentierung immer stärker auch Ton und Argumentation des literarischen Urteils. ${ }^{5}$

Damit hat Michaelis zweifellos Recht. Autonom-ästhetische Wertungskriterien treten in den Hintergrund, die meisten Rezensenten können kaum verhehlen, dass sie den Text nicht als autonomes Kunstwerk, sondern entlang ideologisch aufgeladener Fronten in direkter Abhängigkeit von der öffentlichen Person des Autors bewerten. Auch die erste Rezeptionsphase der Blechtrommel ist nicht völlig frei von autorfokussierten Lesarten, vielmehr wird hier die Autorimago von Grass als fabulierender, unbändiger und etwas exotischer Autor generiert. Doch wird der noch nicht arrivierte Autor Grass an seinem Debütroman gemessen, während in der späteren Rezeption die Texte an ihrem Autor als Person des öffentlichen Lebens, als Intellektueller und als Wahlkämpfer für die SPD gemessen werden.

\section{Vier Rezeptionsphasen}

Das Grass'sche Werk wurde schon sehr zeitnah Gegenstand der Rezeptionsforschung, insbesondere Die Blechtrommel. Bereits neun Jahre nach Erscheinen legt Gert Loschütz seine Dokumentation Von Buch zu Buch - Günter Grass in der Kritik ${ }^{6}$ vor, 1984 stellt Franz Josef Görtz in ,Die Blechtrommel'. Attraktion und $\ddot{A}$ rgernis $^{7}$ Rezensionen aus zwei Jahrzehnten zusammen. Diese frühe Auseinandersetzung mit der Rezeption ist ungewöhnlich und zeigt die dem

\footnotetext{
5 Arnold (1988) 121.

6 Loschütz (1968).

7 Görtz (1984).
} 
Roman unmittelbar beigemessene Bedeutung. Beide Publikationen erschienen allerdings im Luchterhand-Verlag, der auch für Die Blechtrommel verantwortlich zeichnete - insofern handelt es sich also nicht nur um eine Würdigung der Kanonisierung, sondern auch um ein dieselbe reproduzierendes Instrument. Nichtsdestotrotz erweisen sich beide Dokumentationen als Materialgrundlage von unschätzbarem Wert für eine Analyse der Rezeption der Blechtrommel. Für den vorliegenden Beitrag ausgewertet wurden außerdem die Bestände des Innsbrucker Zeitungsarchivs zur deutsch- und fremdsprachigen Literatur ${ }^{8}$, das seit 1960 ca. 120 Artikel zum Roman in der Tages- und Wochenpresse und ca. 70 zur Verfilmung von Volker Schlöndorff von 1979 archiviert hat. Bis heute gibt es eine Vielzahl rezeptionswissenschaftlicher Arbeiten, sowohl zur deutschsprachigen als auch zur internationalen Rezeption, etwa in Schweden, Polen und den Vereinigten Staaten, was wiederum Grass' Kanonisierung in der Weltliteratur belegt.

Vor seinem Debütroman war Grass schon als Lyriker und Dramatiker in Erscheinung getreten und daher im Literaturbetrieb kein völlig Unbekannter. Der Preis der Gruppe 47, der Grass für eine Lesung aus dem Manuskript der Blechtrommel 1958 zugesprochen wurde, sicherte dem Text schon vor Erscheinen große Aufmerksamkeit. Kein deutschsprachiger Roman des 20. Jahrhunderts wurde öfter besprochen als Die Blechtrommel, und das über einen Zeitraum von mehr als 50 Jahren. Mithalten kann da nur Thomas Manns 1900 erschienenes Familienepos Buddenbrooks, auch wenn dieses aus literatursoziologischer Sicht stärker im 19. als im 20. Jahrhundert verankert ist. Was Mann für die erste Hälfte des 20. Jahrhunderts war, ist Grass für die zweite, er ist „das literarische Wappentier der Republik“9.

Eine Grobsortierung der Artikel nach Publikationsdatum lässt vier Phasen erkennen, in denen Die Blechtrommel jeweils intensiv rezipiert wurde, die aber auch als Zäsuren oder zumindest Modifikationen in der Rezeption gelesen werden können. Die erste und naturgemäß intensivste Phase beginnt mit Erscheinen 1959 und zieht sich bis Ende 1960, als Grass zunächst der Literaturpreis der Stadt Bremen zuerkannt, aufgrund der Verweigerung des Bremer Senats aber nicht verliehen wurde. Die zweite breite Auseinandersetzung, in der deutlich wird, dass der Roman bereits widerspruchslos zu den kanonisierten Werken der deutschsprachigen Literatur und darüber hinaus gezählt wird, setzt 1979 mit der gleichnamigen SchlöndorffVerfilmung ein. Weitere 20 Jahre später geht Grass durch die Verleihung des Literaturnobelpreises 1999 in den literarischen Olymp ein, eine Auszeichnung,

8 Innsbrucker Zeitungsarchiv: <http://www.uibk.ac.at/iza/>.

9 Görtz (1984) 17. 
die, entgegen der offiziellen Begründung des Nobelpreiskomitees, als Triumph der Blechtrommel zelebriert wird. 2009 schließlich wird feierlich das 50-jährige Jubiläum begangen. Die vier Phasen bezeugen nicht nur die beständige Re-Lektüre des Textes, sie lassen auch eine Rezeptionsdynamik erkennen, welche über einen simplen Kanonisierungsprozess hinausgeht. Wie jeder Text entgeht auch die Blechtrommel nicht der voranschreitenden Historisierung. Allerdings bleibt nicht jedes literarische Werk so lange Gegenstand öffentlicher Wertungsprozesse. Die Rezeptionsgeschichte des Romans spiegelt daher neben gesellschaftpolitischen Veränderungen auch die Modifizierungen und Umbrüche literaturkritischer Zugänge und Wertungskriterien.

\section{Rezeption bei Erscheinen}

Wieviele Besprechungen genau nach der Veröffentlichung 1959/6o erschienen sind, lässt sich nicht mehr eruieren. Sicher ist, dass Die Blechtrommel in der deutschsprachigen Tages- und Wochenpresse für einen Debütroman extrem breit rezipiert wurde, was auch aus der Verleihung des Preises der Gruppe 47 resultiert - die Kritiker warteten daher schon darauf, ob der Text diesen Vorschusslorbeeren gerecht werden würde. Alle bedeutenden überregionalen Blätter lieferten umfangreiche Besprechungen und auch in einer Vielzahl regionaler und kleinerer Zeitungen erschienen ausführliche Rezensionen, sogar in Publikationen von Interessensverbänden, die üblicherweise nicht das Geschehen im Literaturbetrieb verfolgen, von der Neuen ApothekenIllustrierten über die Unabhängige Baumeister-Zeitung bis zur Zeitschrift für Versicherungswesen. Allerdings haben diese wenig Substanz und reihen, wie Franz Josef Görtz zusammenfassend feststellt, ,lediglich Platitüden ${ }^{10}$ aneinander. Dennoch sind sie ein Indiz für die dem Roman zuerkannte, über das literarische Feld hinausgehende Bedeutung. Görtz legte auch eine empirische Analyse der Rezeption der Danziger Trilogie sowie des Romans Örtlich betäubt von 1959-1969 vor. ${ }^{11}$ Mittels quantitativer Methoden lassen sich "charakteristische Übereinstimmungen im Sprachmaterial ${ }^{\text {“12 }}$ der Rezensionen konstatieren, wofür mehrere Ursachen auszumachen sind. Anders als im aktuellen, von den neuen Medien geprägten Rezensionswesen lagen die Besprechungen in den einzelnen Zeitungen zur Zeit des Erscheinens der Blechtrommel meist Wochen, oft sogar Monate auseinander. In Retrospektive erkennt man einen

\footnotetext{
10 Görtz (1984) 13.

11 Görtz (1978).

12 Görtz (1978) 210.
} 
ungewöhnlich in die Länge gezogenen Rezeptionsprozess, der über ein Jahr andauert. Diese zeitliche Distanz ermöglicht es den Kritikern, sich nicht nur zum Werk selbst zu äußern, sondern auch Stellung zur Rezeption zu beziehen und die eigene Person damit deutlich zu positionieren - die Kritik der Kritik, ein gängiges Instrument der Selbstinszenierung des Kritikers. Übereinstimmungen entstehen zum einen durch die direkte Übernahme von Textpassagen aus anderen Rezensionen, was im behandelten Zeitraum von 1959-1969 weniger leicht auffällt als im Zeitalter von Online-Zeitungen, zum anderen bürgert sich eine Vielzahl immer wieder aufgenommener Begriffe ein, die sich bis zu Stereotypen verfestigen, sowohl für den Autor als auch für den Text (u. a. pornographisch, ekelhaft, nihilistisch, obszön). Zudem lassen sich zwei oppositionelle Positionen eruieren, die in „Anleihen“ beim Vokabular der favorisierten, häufig als Autorität herbeizitierten Leitfiguren“13 münden.

Nach der kargen, den Kahlschlag verkündenden Literatur nach 1945 nahmen Teile der Kritik Grass' kraftvollen, ironischen, mit phantastischen Elementen gespickten Text mit einem aufatmenden Seufzer entgegen. Die Blechtrommel läutet eine neue Phase der deutschsprachigen Nachkriegsliteratur ein: Es darf wieder erzählt werden. Dieses epische Moment in Zeiten des hegemonialen Kahlschlags ist auch Grundlage der affirmativen Aufnahme im Feuilleton. Doch es gibt nicht nur Lobeshymnen, ganz im Gegenteil: Dass Die Blechtrommel der bedeutendste deutsche Roman der Nachkriegszeit in Deutschland und international werden sollte, lässt sich an den Kritiken nicht ablesen. Der Text fasste keineswegs nur Lob aus, wie es etwa der bekannteste deutschsprachige Literaturkritiker Marcel Reich-Ranicki in seiner Rezension in der Zeit vom 1.1.196o darstellte. Insofern positioniert sich Reich-Ranicki oppositionell zu einem Hype, den es so gar nicht gibt, eine häufig angewendete Strategie in Wertungsdiskursen: Der Verweis auf eine vermeintlich kollektiv geteilte Haltung der Kritik zu einem Text, um sich selbst in inszenatorischer Absicht davon abzugrenzen. So spricht er vom „Enthusiasmus fast der gesamten deutschen Kritik" ${ }^{\text {“4 }}$, während Grass für ihn selbst ,vorläufig noch keineswegs ein guter Erzähler“ sei. Sein Urteil fällt ausgesprochen harsch aus: „Wäre der Roman um mindestens zweihundert Seiten kürzer, er wäre - wenn auch sicher kein bedeutendes Werk - doch weit besser." Allerdings konnte und wollte Reich-Ranicki diese Kritik nicht lange aufrecht erhalten. Schon 1963 revidiert er sein eigenes Urteil in einer Radiosendung im Westdeutschen Rundfunk. In der Sendung mit dem Titel "Selbstkritik eines Kritikers“ hält er zwar manche Kritikpunkte aufrecht, möchte aber anders gewichten und erinnert sich auch

13 Görtz (1978) 232-233.

14 Marcel Reich-Ranicki, Die Zeit, 01.01.196o, zitiert nach Görtz (1984) 117. 
etwas widersprüchlich: So meint er, drei Jahre vorher schon von einem ,überdurchschnittlichen Erzähler gesprochen zu haben, während genau das Gegenteil der Fall war. Die öffentliche Revision seines Urteils dokumentiert indes den Stellenwert, den Grass' Roman schon wenige Jahre nach Erscheinen errungen hatte. Andere erkannten gleich, dass hier ein Jahrhundertwerk vorlag, so wagt es der Evangelische Literaturbeobachter „zu behaupten, daß von der jungen Generation nach dem Zusammenbruch, ja, ohne Günter Grass mit Thomas Mann vergleichen zu wollen, seit den Buddenbrooks kein gewichtigeres episches Gebilde vorgelegt worden ist als Die Blechtrommel." ${ }^{15}$ Der damals noch weitgehend unbekannte Hans Magnus Enzensberger verglich den Roman mit Döblins Berlin Alexanderplatz und Brechts Baal und prophezeite, es handle sich um ein Werk, „an dem Rezensenten und Philologen mindestens ein Jahrzehnt zu würgen haben, bis es reif zur Kanonisation oder zum Vergessenwerden ist." ${ }^{\prime 16}$

\section{Moralisierende Wertung}

Das Erscheinen der Blechtrommel fällt in die von konservativen, christlichen Werten, dem Vorgehen gegen Kommunismus und Sozialdemokratie und der Relativierung des Nationalsozialismus geprägte Adenauer-Ära.Jürgen Matheny beschreibt treffend: „Die Kritik der ersten Stunde hat gespürt, mehr gespürt als erkannt, daß von hier eine ,Epoche‘ in der Nachkriegsliteratur ausging, wenn sie dies auch mit einem schiefen Vokabular beschrieben, mit falschen Akzenten versehen und widersprüchlichen Aussagen belegt hat."17

Die Bewertungen fallen weit unterschiedlicher aus, als das in späteren Rezeptionsphasen, etwa nach Verleihung des Nobelpreises für Literatur 1999, dargestellt wird. Die nicht wenigen Verrisse legen - wiewohl sie es gerne suggerieren - nicht literarisch-ästhetische, sondern moralische Maßstäbe an die Geschichte des kleinwüchsigen Blechtrommlers Oskar Mazerath an. Das zeigen auch die von Görtz herausgearbeiteten Stereotype. Peter Hornung bezeichnet den Roman in der Deutschen Tagespost als ,epileptische Kapriole und urteilt weiter: „Grunzend kann ich nur das Behagen nennen, mit dem Grass in Abnormitäten und Scheußlichkeiten wühlt. Konsequent macht er

\footnotetext{
15 Hlg [Kürzel nicht mehr auflösbar], Evangelischer Literaturbeobachter, Dezember 1959, zitiert nach Görtz (1984) 90.

16 Hans Magnus Enzensberger, Süddeutscher Rundfunk, 18.11.1959, zitiert nach Görtz (1984) 62 .

$17 \quad$ Arnold (1988) 24 .
} 
sich über jeden moralischen und ethischen Anspruch lustig. Vom Religiösen ganz zu schweigen. ${ }^{“ 18}$ Hornung nimmt den Text für bare Münze und kritisiert in empörten Tonfall die absente Moral Oskars, die er zur moralische Bankrotterklärung der Gruppe 47 analog setzt und die er persönlich angreift: „Ich gebe ja zu, daß das fortwährende Unterschreiben von Manifesten gegen die Atombewaffnung der Bundeswehr den Geist eintrübt." Hornungs Verriss ist ein paradigmatisches Beispiel, sowohl für den sprachlichen Duktus als auch für die Bewertungsschemata, die in der ersten Rezeptionsphase zum Tragen kommen:Die Kontroversen rund um Die Blechtrommel führen entlang ideologischer, politischer und moralischer Fronten und entzünden sich keineswegs an ästhetischen und formalen Fragestellungen. „Von der Kirchenschändung bis zur allerübelsten Pornographie, es wird alles dargeboten, was verletzen kann" ${ }^{419}$, urteilt Unser Danzig.

Die damals sehr einflussreiche evangelisch-konservative Zeitung Christ und Welt gibt sich naturgemäß besonders schockiert, und befürchtet die Verschleierung des unsittlichen Inhalts gerade durch die ästhetische Qualität:

Man liest das Buch beinahe schlechten Gewissens. Das ist alles in einem Ausmaß ,gekonnt', das Ergreifendste und Frevlerischste so elegant entpathetisiert, der Mensch so restlos ,fertig'-gemacht, daß man von der ästhetischen Vollendung (von einigen indiskutablen Ferkeleien abgesehen) versucht ist, sittliche Argumente als unangebracht beiseitezuschieben. ${ }^{20}$

Angegriffen wird auch der Autor persönlich, das Buch „verrät den anomalen Hang des Autors zum Ekligen“, was Christ und Welt als Generationenproblem der in den vierziger Jahren sozialisierten Jugend zu entlarven meint.

Vornehmlich zwei Bereiche kommen in der moralisierend-engagierten Literaturkritik zum Tragen: Sexualität und Religion. Insbesondere stoßen sich Kritiker an der Szene, in der Oskar in der Kirche mit einer Jesusfigur kommuniziert, ja in Konkurrenz tritt, was als ,Blasphemie‘ und ,Verhöhnung Christi ${ }^{21}$ angeprangert wird. Auch das extremste Beispiel von Kritik hat die Blechtrommel

18 Peter Hornung, Deutsche Tagespost, 23./24.11.1959, zitiert nach Loschütz (1968) 24-25.

19 K. [Kürzel nicht mehr auflösbar], Unser Danzig, 20.05.196o, zitiert nach Loschütz (1968) 26.

20 Werner Wien, „Trauermarsch auf der Blechtrommel“, Christ und Welt, 17.12.1959, [So nicht anders angegeben, sind die Artikel zitiert nach dem Innsbrucker Zeitungsarchiv, das für seine Altbestände leider keine Seitenangeben zur Verfügung stellt.]

H. Müller-Eckhard, Kölnische Rundschau, 13.12.1959, zitiert nach Görtz (1984) 96-99. 
hervorgerufen: „Wir warnen in aller Form davor, dieses Machwerk zu empfehlen oder Menschen mit unverdorbenem Geschmack in die Hand zu geben.“22 Günter Blöcker wirft Grass hingegen eine ,unappetitliche[...] l'art pour l'art'Gesinnung vor, die das Grauen nicht in Katharsis verwandle, sondern sich an der Erniedrigung labe: „So hinterläßt das überfüllte Buch am Ende den Eindruck einer wahrhaft gräßlichen Leere. In seinem konsequent antihumanen Klima gibt es nur eines, woran man sich halten kann: den Selbsthaß““23

Die positiven Rezensionen hingegen nehmen vor allem das erzählerische Moment in den Fokus und lesen den Roman allegorisch als überfälligen Protest. Betont werden der Einfallsreichtum und die kraftvolle Sprache. Dabei fällt es schwer, dieses Erzählen genauer zu beschreiben, die Kritiker verfallen immer wieder in dieselben Plattitüden: wild, prall, überschäumend, wuchtig. Außerdem kristallisiert sich schon jetzt heraus, was die Rezeption der Blechtrommel bis heute am meisten beschäftigt: ihr Protagonist. Der glaszersingende, blechtrommelnde Danziger Oskar Matzerath, der mit drei sein Wachstum einstellt und als Insasse einer Heilanstalt die unzuverlässige Erzählinstanz darstellt, ruft als Figur extreme Gefühle hervor:

Sogar am Tod der Mutter ist dieser verderbte Giftzwerg beteiligt. An Widerwärtigkeit diesen Verbrechen ebenbürtig sind die Amouren des Gnoms, die mit einem Abenteuer mit der späteren Stiefmutter beginnen und sich zu einem Kreszendo des Absurden und Abstoßenden steigern. ${ }^{24}$

Das Beispiel zeigt, wie sich die konservativ-moralisierende Literaturkritik zu einer identifikatorischen, realistischen Lesart hinreisen lässt, die nicht nur einer Figur ihr Handeln vorwirft, sondern dieses auch noch ihrem Autor anlastet. Keine Rede vom Nationalsozialismus, keine Rede davon, wofür die Figur des infantilen Blechtrommlers eigentlich stehen könnte, stattdessen dominiert eine Diktion und ein Kunstverständnis, das den Geist der Vorstellung von einer entarteten Kunst immer noch in sich trägt. Nimmt man die Rezeption der Blechtrommel als Paradigma der deutschsprachigen Literaturkritik der 1950er und 6oer Jahre, so lässt sich konstatieren, dass solche Lesarten keine Ausnahmen sind. In der zweiten Rezeptionsphase, Ende der 1970er spielen diese keine Rolle mehr. Heute ruft ein solches Vorgehen Proteststürme hervor: Als der Kritiker Georg Diez 2012 Christian Kracht des Antisemitismus bezichtigte und das auf Basis seiner Romanfigur August Engelhardt aus dem Roman

22 H. Müller-Eckhard, Kölnische Rundschau, 13.12.1959, zitiert nach Görtz (1984) 96-99.

23 Günter Blöcker, FAZ, 28.11.1959, zitiert nach Görtz (1984) 75 .

24 Hornung, zitiert nach Görtz (1984) 51. 
Imperium argumentierte, stellte sich das Feuilleton ungewohnt geschlossen hinter Kracht und erklärte damit biografisierende Rückschlüsse vom Text auf den Autor für illegitim, während dasselbe Vorgehen 1959 nicht problematisiert wurde.

\section{Die Blechtrommel als Maßstab}

Sieht man sich die Besprechungen der Folgewerke von Grass an, so fällt auf, dass Die Blechtrommel schon ab der folgenden Novelle Katz und Maus dauerhaft als Maßstab herangezogen wird. 1961, also nur zwei Jahre nach seinem Erscheinen, wird die Blechtrommel wie ein Klassiker behandelt: Die Kenntnis des Inhalts wird vorausgesetzt, die literarische Bedeutung des Werkes, egal ob der jeweilige Kritiker positiv oder negativ dazu steht, ist außer Zweifel. Die Rezensionen zu Katz undMaus lesen sich wie komparatistische Fingerübungen:

Statt mehr als siebenhundert Seiten knapp hundertundachtzig; statt eines breit ausladenden, immer neue Verrücktheiten gebärdenden Geschehens ein, wenn auch nicht gerade karger, so doch begrenzter Anfall von Ereignissen; statt des bösen trommelnden Zwerges Oskar ein Held, der lediglich einen überdimensionalen Adamsapfel aufzuweisen hat - man sieht, bei Günter Grass haben sich die Verhältnisse beträchtlich normalisiert. Zum Vorteil? Haben wir eine Einbuße zu beklagen?25

Alle ausgewerteten Besprechungen der Novelle verfahren nach diesem Vergleichsverfahren, das auch bei späteren Texten noch zum Einsatz kommt.

War Marcel Reich-Ranickis zunächst negatives Urteil noch nicht prägend für die Rezeption der Blechtrommel, so dominierte seine Meinung sehr bald die feuilletonistische Aufnahme und Einschätzung der späteren Werke. Deutschlands Großkritiker und Deutschlands in der öffentlichen Wahrnehmung bedeutendster Autor blieben über Jahrzehnte in einer, wie Grass es ironisch bezeichnete, ,Zwangsehe ${ }^{26}$ verbunden. Aus der Zwangsehe entspann sich spätestens mit dem in Form eines offenen Briefes verfassten Verrisses von Ein weites Feld 1995 (das Bild auf dem Cover des Spiegel von Reich-Ranicki beim physischen Zerreißen des Buches war indes eine Fotomontage) eine Fehde, die bis zum Tod des Kritikers 2013 von beiden Seiten leidenschaftlich gepflegt wurde. Obwohl zunächst verrissen, installierte auch Reich-Ranicki in

25 Jost Nolte, Die Welt, 19.10.1961, zitiert nach Loschütz (1968) 31.

26 Arnold (1988). 
späteren Besprechungen die Blechtrommel als Maßstab für alle Folgetexte, die an den Debütroman nicht anschließen konnten.

Die weitere Rezeptionsdynamik des Grass'schen Werkes ist ein Paradoxon: Gerade mäßig begeisterte Rezensionen oder sogar Verrisse waren dazu angetan, den Ruhm der Blechtrommel bis zur Verklärung hin zu erhöhen, was dazu führte, dass der Text selbst mittlerweile vom Objekt der Kritik zum verabsolutierten Wertungskriterium avancierte - die Blechtrommel wird zur Chiffre für den perfekten Roman, der naturgemäß niemals übertroffen werden kann. Schon zehn Jahre später gilt Grass als Institution, die sich selbst nicht mehr das Wasser reichen kann: „Was ist aus Günter Grass geworden, zehn Jahre nach dem weltweit vernommenen Blechtrommel-Wirbel?" 27 fragt die FAZ. Das Erscheinen des Romans Örtlich betäubt 1969 wird von heftigen Verrissen begleitet. Kraftlos, gezähmt, am absteigenden Ast, so präsentiert die Kritik Deutschlands bekanntesten Autor. Das Urteil: „Örtlich betäubt ist nicht Die Blechtrommel. Im Vergleich mit den früheren Büchern ist der neue Roman von vornherein verloren." ${ }^{28}$ Auch Jahrzehnte und mehr als zehn Prosatexte sowie mehrere Lyrikbände und Dramen später ist es immer noch Die Blechtrommel, die gerade bei negativen Besprechungen herangezogen wird. So meint etwa Christian Thomas in der Frankfurter Rundschau:

Die Diskrepanz zwischen dem Erzähler Grass und dem Verlautbarungslyriker (und Leitartikelkonkurrenten) könnte nicht krasser sein. Wenn wir etwa über Die Blechtrommel sprechen, dann über sarkastisch gebrochene Einfühlungstechniken. Ihnen ist kein Wort im politischen Handgemenge gewachsen, und der erste, der dies wissen müsste, ist, wer denn sonst, Günter Grass. ${ }^{29}$

Die Blechtrommel wird gar zum ,Grass-O-Meter der deutschen Großkritik ${ }^{30}$ hochstilisiert. Literaturwissenschaftlich betrachtet machen es sich viele Rezipienten späterer Werke sehr einfach, indem sie dem jeweils negativ besprochenen Text bescheinigen, nicht über die Qualität des Debütromans zu verfügen und die Mängel mit Gemeinplätzen füllen, wie etwa im Rheinischen Merkur: „Es war, als ginge ihm die Sprache aus, mit der er sich in ungeahnte Höhen verstiegen hatte. Sie war längst bemüht und angestrengt und hatte nichts mehr von der Eleganz und zwingenden Überzeugungskraft der Blech-

\footnotetext{
27 Segebrecht (1969).

28 Segebrecht (1969).

29 Thomas (2012).

30 Butterweck (1999).
} 
trommel “31 schreibt Volker Wieckhorst anlässlich der Nobelpreisverleihung über einen „Günter Grass, der schon Geschichte ist“.

\section{Re-Lektüre durch die Verfilmung}

Dass der Roman über 50 Jahre beständig in der Diskussion blieb, hat auch mit der nicht weniger kontroversiell aufgenommenen Verfilmung von Volker Schlöndorff zu tun, die 1979 die Goldene Palme in Cannes erringen konnte und 1980 als bester ausländischer Film mit einem Oscar prämiert wurde. Schon die Ankündigung der Verfilmung reaktivierte das Interesse am literarischen Prätext. Literaturverfilmungen haben beinah immer das Los, an der literarischen Vorlage gemessen zu werden. Dabei wird eine breit angelegte Re-Lektüre des Prätextes initiiert, anhand derer auch Konstanten und Brüche in der Rezeption sichtbar werden. Bei in Vergessenheit geratenen Texten wird etwa nur kurz auf die literarische Vorlage verwiesen.

Verfilmungen von kanonisierten Werken enden hingegen meist in Doppelbesprechungen, bei der der literarische Text automatisch als Wertmaßstab gesetzt wird. Auf diese Weise wird einerseits die Eigenständigkeit des Filmes als Kunstwerk unterwandert, andererseits gleichzeitig der Kanonisierungsgrad der Vorlage erhöht. So auch im Fall der Blechtrommel: Eine Analyse der Filmkritiken zu Schlöndorffs Blechtrommel in Hinblick auf Grass' Vorlage liefert vor allem eine Erkenntnis, nämlich dass der Prätext innerhalb von zwanzig Jahren einen intensiven Prozess der Kanonisierung durchlaufen hat. Der ambivalente Tenor der Kritik von 1959/6o ist 1979 einer kollektiven, beinahe stillschweigenden Affirmation gewichen: Die Qualität der Blechtrommel steht nicht mehr zur Disposition. „Mehr ein Mythos als ein Buch“ und ,eine nationale Legende“ sei diese mittlerweile, „eine ganze Generation von Deutschen“ habe sich mit dem Roman „identifiziert“32. Es gibt nur ganz wenige Ausnahmen, etwa wenn Ulrich Greiner in der FAZ nach seiner Re-Lektüre des Romans festhält, es sei „sicher kein Jahrhundertwerk, kein revolutionäres Epos“33. Mit dieser Meinung steht Greiner allerdings allein da. Die anderen Besprechungen kritisieren meist den Film, weil dieser zwar nahe an der literarischen Vorlage bleibe, es aber nicht schaffe, dessen künstlerische Originalität ins audiovisuelle Medium zu übersetzen.

\footnotetext{
$31 \quad$ Wieckhorst (1999).

32 Ignée (1979).

33 Greiner (1979).
} 
Als repräsentatives Beispiel für die Tendenz der Besprechungen in Bezug auf Grass' Blechtrommel kann die Besprechung Sigrid Löfflers, einer der renommiertesten deutschsprachigen Literaturkritikerinnen, im Profil vom 20.08.1979 gelten. Zunächst wird die Bedeutung der literarischen Vorlage gewürdigt:

Heute ist Die Blechtrommel längst kanonisiert. Sie ist der erste Klassiker der deutschen Nachkriegsliteratur, sie ist ein Welterfolg mit einer Gesamtauflage von knapp drei Millionen Exemplaren und Übersetzungen in 20 Sprachen, sie ist Dissertationsgegenstand und seit langem beigesetzt in nichts als Literaturgeschichte. ${ }^{34}$

Was 1959 ein Skandal war, „kratzt keinen Pfaffen und keinen Heimatvertriebenen mehr. ${ }^{\text {“5 }}$ Negative Besprechungen von Schlöndorffs Film werden mithin zu Leseempfehlungen für Grass: „Spätestens nach Ende der 150 Minuten brav bemühter Literaturbebilderung wird einem klar: Man sollte wieder einmal Die Blechtrommel von Günter Grass lesen." Die Verfilmung wird auch zum Anlass genommen, die Rezeption des Romans unmittelbarnach seinem Erscheinen auf ihren Bestand hin zu überprüfen. Aus der retrospektiv olympischen Position, die über das Wissen um die vorangeschrittene Kanonisierung des Grass'schen Debütromans verfügt, werden vor allem die Kritiker negativer Besprechungen regelrecht vorgeführt und süffisant ihr nun offenkundiges Fehlurteil seziert. Freilich hat in der Zeit eine Verschiebung moralischer Grenzen stattgefunden, von der die Literatur stärker profitierte als der Film; was gesagt werden darf, darf man noch lange nicht zeigen. Am Buch gelobt und hervorgehoben wird jetzt vor allem das, was die Verfilmung nach Meinung der Kritiker - fast ausschließlich Literatur- und keine ausgewiesenen Filmkritiker - nicht umzusetzen vermag: die Vielschichtigkeit und Polyvalenz, die pikareske Perspektive Oskars, die, auch so ein Wort, das für Grass' Sprache ständig verwendet wird, ,barocke‘ Kraft und Radikalität seiner Bilder.

\section{Literaturnobelpreis}

Als Triumph der Blechtrommel - so wurde der Literaturnobelpreis für Günter Grass größtenteils rezipiert, in offenkundiger Missachtung der offiziellen Begründung des Nobelpreis-Komitees. Dieses bezeichnet zwar den Debütroman als bleibendes Werk der Weltliteratur, würdigt aber auch das

34 Löffler (1979).

35 Löffler (1979). 
restliche Oeuvre des damals 71-Jährigen, unter anderem das von Reich-Ranicki kampagnenartig vernichtete Ein weites Feld. „Selbst die Nobelpreisverleihung wurde leise murrend mit dem Hinweis kommentiert, sie käme vierzig Jahre zu spät“, konstatiert der Falter die von Hassliebe geprägte Beziehung des Feuilletons zu Deutschlands erfolgreichstem Autor. Dem Urteil des Falters kann man sich indes nur anschließen, wenn man die implizierte Aussage vieler Reaktionen beachtet, die unter viel Lob und Begeisterung zum Vorschein kommt. Reich-Ranicki, der sich sehr erfreut gab, konnte es sich nicht verkneifen anzumerken: „[...] dass die letzten Bücher schlechter sind als sein Frühwerk, spielt dabei keine Rolle.“36

Das ist auch der Tenor der meisten anderen Artikel zum Nobelpreisgewinn: Die Blechtrommel gilt als unerreichtes Meisterwerk: „Er ist einer der letzten Erzähler des Jahrhunderts geblieben - auch wenn das grandiose Meisterwerk, ,Blechtrommel Zwo' sozusagen, nie gekommen ist.“37 „An seine Anfänge ist Günter Grass nie mehr herangekommen“38 meint Anton Thuswaldner in den Salzburger Nachrichten und Ulrich Baron schreibt in der Welt aus, literaturkritischer Sicht': „Was man dem Autor der Danziger Trilogie gerne zugebilligt hätte, mag man dem Autor von Ein weites Feld und Mein Jahrhundert als Kritiker nicht mehr recht zugestehen."39 Am nächsten Tag legt man nochmals nach: „Es gab eine Zeit, da wäre der richtige Zeitpunkt gewesen, ihm den Preis zu verleihen; er ist lange verstrichen. ${ }^{40}$ Auch SchriftstellerkollegInnen feiern in erster Linie den Blechtrommelautor. Kathrin Schmidt: „Die hervorragendsten Bücher des Günter Grass wurden vor vielen Jahren geschrieben. Ich denke, sie sind gemeint." ${ }^{41}$ Damit prolongiert die Kritik unter dem Vorzeichen des Lobes ihre ablehnende Haltung. Als in der öffentlichen Wahrnehmung oberste Konsekrationsinstanz des internationalen Literaturbetriebs schafft die Nobelpreisjury dazu einen Ausgleich: „In der öffentlichen Debatte seines Heimatlands ist er Kraftquell und Fels des Ärgernisses, aber für literarische Größen draußen in der Welt wie García Márquez, Rushdie, Gordimer, Lobo Antunes und Kenzaburo Oe ein bewunderter Vorgänger"42, heißt es in der Urteilsbegründung der Akademie. Die FAZ kommentiert: „Dies ist die äußerste Genugtuung für Günter Grass - dass er, gestützt auf die internationale

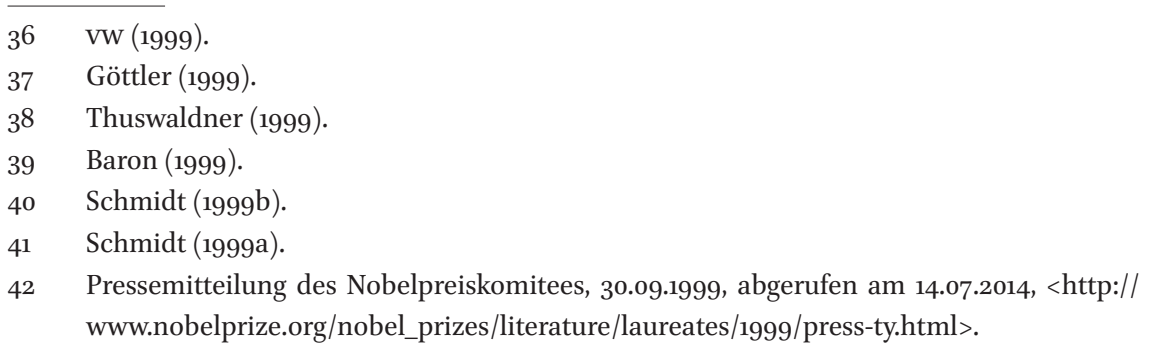


Öffentlichkeit, nun Recht behält gegen die Kritiker im eigenen Lande.“43 Dabei kommen diese Kritiker gerade von Seiten der intellektuellen Rechten und des konservativen Feuilletons. Frank Schirrmachers, Verneigung' vor Grass im Leitartikel der FAZ liest sich wie ein Nachruf, kritisiert Thomas Anz in der Süddeutschen Zeitung. ${ }^{44}$

Der Nobelpreis wurde Grass vor Bekanntwerden seiner Mitgliedschaft in der Waffen-ss verliehen und vor dem Skandalgedicht ,Was gesagt werden muss', doch schon vorher leidet Grass' Reputation. Während politisches Engagement goutiert, wenn nicht gar gefordert wird, ist offenes parteipolitisches Auftreten nicht gerne gesehen. ,Der Es-Pe-De-Trommler' wird Grass sarkastisch genannt und auch für seine Haltung zur DDR bleibt Kritik nicht aus.

Die Verleihung des Literaturnobelpreises initiiert einen Revisionsprozess der bisherigen Rezeption des Schriftstellers Grass. Aus literaturwissenschaftlicher Sicht fällt vor allem die oppositionelle Bewertung von öffentlichem Wirken und Werk auf. Der Intellektuelle Grass schiebt sich in der Rezeption immer mehr vor sein Werk:

Seit vielen Jahren wurde Grass in Deutschland kaum noch als Schriftsteller wahrgenommen, sondern als Prototyp des sich einmischenden Intellektuellen, als eine gesellschaftliche Instanz. Diese Rezeptionsweise hat Grass selbst gesucht [...] und das prägte auch immer die Diskussion um seine literarischen Texte. Daß sie in zunehmendem Maße von der professionellen Kritik verrissen wurden, war vorhersehbar, entwickelte aber ein merkwürdig dialektisches Verhältnis zum Verkaufserfolg. ${ }^{45}$

Dabei wird auf einige wenige Prosaexte von Grass referiert, denen in stereotypischer Manier spezifische Wertigkeiten zugesprochen werden, ohne diese zu benennen: Die Blechtrommel ragt unantastbar aus der gesamten Nachkriegsliteratur heraus, sie wird als das bedeutendste Werk der Epoche vorausgesetzt; den anderen Pol markiert Ein weites Feld, dessen Inferiorität wiederum außer Frage zu stehen scheint. Das restliche Oeuvre wird kaum angetastet, Der Butt manchmal erwähnt, ansonsten höchstens noch auf die Danziger Trilogie verwiesen, die allerdings größtenteils synonym für den ersten Teil verwendet wird. Grass als Vertreter der Dramatik und Lyrik, also jener Genres, aus denen er eigentlich kommt, wird überhaupt nicht gewürdigt. Eindeutig ist: Keines der späteren Werke von Grass kann sich ansatzweise mit

\footnotetext{
43 Steinfeld (1999).

44 Anz (1999).

45 Böttiger (1999).
} 
seinem ersten Roman messen. Was die erneute Rezeption der Blechtrommel angeht, so lässt sich ein nostalgischer Grundton heraushören: „Man kann noch heute, 40 Jahre nach dem Erscheinen der Blechtrommel, irgendeine Seite dieses Romanes [sic] aufschlagen und merkt sogleich: Hier erzählt einer nicht bloss, hier hat einer Sprache, nahrhaftes Vollkorn, neben dem so vieles wirkt wie ungetoasteter Toast. ${ }^{46}$ Dass eben jener unantastbare Roman bei seinem Erscheinen äußerst umstritten war, hat im kollektiven Gedächtnis des Feuilletons keinen Eingang gefunden. Vielmehr will man sich daran erinnern, dass „Die Blechtrommel durchgehend als erzählerisches Meisterwerk anerkannt wurde“ ${ }^{47}$ Das Lob der Blechtrommel hat sich auf wenige, mantraartig wiederholte Zuschreibungen reduziert, die in beinahe allen Artikeln zum Nobelpreis auftauchen.

\section{0-jähriges Jubiläum}

SchriftstellerInnenjubiläen sind häufig; dass das Jubiläum eines literarischen Werkes begangen wird, ist selten und wird nur den ganz großen Texten zuteil. Im Günter-Grass-Haus in Lübeck findet die Ausstellung, 50 Jahre Blechtrommel statt, es gibt einen großen Festakt mit Bundeskanzler a.D. Gerhard Schröder als Festredner, das Feuilleton widmet sich ausführlich dem Werk und seiner Wirkungsgeschichte. Anders als in der Berichterstattung zum Nobelpreis kommt tatsächlich Begeisterung auf: Gefeiert wird der Text; sein Schöpfer und dessen späterer Werdegang als streitbarer Intellektueller werden ausgeblendet. Je weiter Die Blechtrommel in die Vergangenheit rückt, umso größer die Idealisierung und Stereotypisierung der Werturteile. Die zunächst noch sehr divergierenden Meinungen sind einer intersubjektiven, kollektivierbaren Lesart gewichen: Die Figur des Oskar Matzerath wird allegorisch als Protest gegen den kleinbürgerlichen Mief und die prolongierte nationalsozialistische Gesinnung der Nachkriegsjahre in Deutschland gelesen. Wenige Szenen bleiben haften und werden in beinahe allen Beiträgen zum 5o-jährigen Jubiläum wieder angesprochen, wobei auffällt, dass die Erinnerung stark von Schlöndorffs Verfilmung geprägt ist, etwa der Pferdekopf mit den Aalen, Oskar trommelnd unter der Tribüne und die Brausepulver-Szene. Die wenigen tatsächlich analytischen Besprechungen aus der ersten Rezeptionsphase, etwa von Hans Magnus Enzensberger, Walter Höllerer und Joachim Kaiser, haben sich schlussendlich durchgesetzt. Einig ist man sich im Rückblick auch, „mit Oskar

$46 \quad$ Isenschmid (1999).

47 N.N. (1999). 
Matzerath kehrte 1959 die deutsche Nachkriegsliteratur auf die Weltbühne zurück. ${ }^{48}$ Neben dem wiederholten Lob für die kraftvolle Sprache, die zersplitterte Erzählperspektive, die subversive Ästhetik, wird nun vor allem die historische Bedeutung im Kontext der Vergangenheitsbewältigung betont: „Lesen wir heute Die Blechtrommel als Zeugnis einer Teilhabe am Zeitgeschehen, so ist das Aufzeigen des Grauens gnadenlos und trotzig und mutig: Wirft die vor Eigensinn strotzende Darstellung des Infantilen, der sich selbst des Mordes beschuldigt, nicht eine heikle und allzeit gültige Frage auf? Die Frage, wie viel Schuld der Einzelne auf sich geladen hat, wie viel Verantwortung er tragen kann und muss?"49 Die vielen meist sehr ausführlichen Besprechungen anlässlich des Jubiläums stammen von einer neuen Generation von Kritikern und Kritikerinnen, den ,Nachgeborenen ${ }^{50}$, die sowohl in der inhaltlichen als auch in der formalen Bewertung der Blechtrommel völlig neue Voraussetzungen mitbringt. Immer wieder ins Feld geführt werden die Jahrgangskollegen von 1959, Uwe Johnsons Mutmaßungen über Jakob und Heinrich Bölls Billard um halb zehn. Beide hatten bei Erscheinen ebenfalls für Aufruhr gesorgt, dauerhaft kanonisiert wurde aber nur Grass' Roman. Während die anderen heute ,eher Pflichtlektüre' sind, „überkommt einen“ bei der Blechtrommel „die wahre Lust des Lesens ${ }^{\text {"51. }}$.

\section{Internationale Bedeutung der Blechtrommel}

Nach der Zäsur durch den Nationalsozialismus und die Shoa befindet sich die deutsche Literatur in einer Sonderposition: Gebunden an die ideologisch aufgeladene Tätersprache ist sie gezwungen, sich ein neues Selbstverständnis zu erarbeiten; durch die außerordentliche moralische Verantwortung sieht man sich zum ästhetischen Kahlschlag verpflichtet. Frank Schirrmacher spricht von der Stunde Null der westdeutschen Literatur:

Während in der Literatur der Nachbarländer der Abschied von einem stabilen, selbstbewußten Ich längst vollzogen war, begann in Westdeutschland eine junge Literatur damit, es nicht für sich, sondern für eine ganze Nation wiederherzustellen. Dem Ich wurde zugemutet, die

\footnotetext{
48 Dotzauer (2009).

49 Franck (2009).

50 Franck (2009).

$5^{1} \quad$ Wittstock (2009).
} 
durch den einzigartigen historischen Bruch geschlagene Wunde zu heilen. 52

Ohne ihn zu nennen wird der kleinwüchsige Oskar zum Symbol der Erfahrungen der Nachgeborenen auserkoren: „Das Dritte Reich erschien den Nachgeborenen denn auch wie eine böse, aber märchenhaft verzerrte Kindheitserinnerung, in mythischen Vorzeiten angesiedelt und aus aller Geschichte

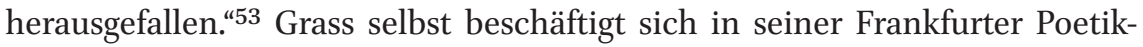
vorlesung von 1989/9o mit Adornos berühmtem Diktum, wonach ein Gedicht nach Auschwitz Barbarei sei und negiert diesen kategorischen Imperativ, dem er sich in der Blechtrommel schon literarisch widersetzt hat. Mit dem ersten Teil seiner Danziger Trilogie setzt er dem Kahlschlag ein Ende, ohne diese Verantwortung zu negieren, ein Vorhaben, das international Anerkennung findet. Es ist tatsächlich Die Blechtrommel, welche die deutschsprachige Literatur wieder auf die Weltbühne zurückbringt. Schon 1961 erschien Le Tambour in Frankreich, mittlerweile liegt der Roman in über 40 Sprachen vor. Weder für Günter Grass noch für das deutschsprachige Feuilleton absehbar war der unmittelbar einsetzende internationale Erfolg des Textes ${ }^{54}$, was sich sowohl in Verkaufszahlen als auch in einer intensiven literaturkritischen Rezeption niederschlug. In manchen Ländern war die Rezeption gar dem Werk selbst voraus. Obwohl Die Blechtrommel erst 1983 auf Polnisch erschien, war Grass in den Medien ungewöhnlich präsent, wobei vor allem seine Herkunft als gebürtiger Danziger und Kaschube und sein politisches Engagement thematisiert wurden. ${ }^{55}$ Der Nobelpreisgewinn wurde schließlich in Polen wie ein Heimsieg gefeiert. Auch in den USA gab es schon vorab großes Interesse, bis der Roman 1963 schließlich auf den amerikanischen Buchmarkt kam. ${ }^{56}$ Sowohl der Autor selbst als auch die professionelle Leserschaft im deutschsprachigen Raum gingen davon aus, es handle sich bei den im Roman verhandelten Themen um spezifisch deutsche, welche für ein internationales Publikum nicht von Interesse seien:

Für mich war die Überraschung, da man ja immer wieder auf momentanen Erfolg anspricht, daß z. B. ein Roman wie Die Blechtrommel nicht nur in Deutschland Interesse und Leser fand, sondern auch im Ausland.

\footnotetext{
$5^{2} \quad$ Schirrmacher (1990).

53 Ebd. Vgl. auch: Roberts (1994) 249.

54 Zur genauen Wirkungsgeschichte vgl.: Neuhaus (2010) 10-14.

55 Vgl.: Gesche (2003).

$5^{6} \quad$ Zur Rezeption in den usA vgl.: Mayer (1979).
} 
Damit hatte ich überhaupt nicht gerechnet, daß sich jemand im Mittleren Westen der Vereinigten Staaten oder in Südfrankreich oder in Skandinavien für kleinbürgerlichen Mief im Übergang von der Weimarer Republik zum Nationalsozialismus etc. interessieren könnte. ${ }^{57}$

Die Selbsteinschätzung des Autors und die Rezeption seines Textes divergieren hier deutlich. So war die amerikanische Rezeption, die insgesamt sehr heterogen ausfiel, sich doch weitgehend einig darüber, es mit „einer längst überfälligen literarischen Objektivierung deutscher Schuld aus deutscher Sicht“ ${ }^{458}$ zu tun zu haben. Damit war sie der deutschsprachigen Kritik deutlich voraus, die dem Aspekt der Schuld in der ersten Rezeptionsphase weniger Bedeutung beimaß.

Etwas widersprüchlich ist, dass die deutschsprachige Kritik zunächst die internationale Relevanz des Textes verkennt, gleichzeitig aber literarisch im europäischen Kontext verortet, vor allem über intertextuelle Bezüge zur karnevalistischen Literatur und zum Schelmenroman. Die pikareske Figur des Oskar Matzerath wird in eine Traditionslinie mit dem Don Quiote, mit den Werken Rabelais' und Jean Pauls gesetzt: „Es ist die Tradition der ironisch und parodistisch gebrochenen Geschichts- und Welterzählung, die aufgetragen ist auf das

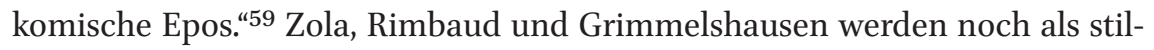
prägend für die Blechtrommel angeführt, auch Swift und Kafka; das politische Engagement sei hingegen im Sinne Sartres. ${ }^{60}$ In der späteren Rezeption wird Die Blechtrommel dann wiederum als literarisches Vorbild für Autoren wie Salman Rushdie und John Irving gesehen.

\section{Resümee}

Die Blechtrommel hat mittlerweile eine Rezeptionsgeschichte von über fünfzig Jahren. Nach der kontroversiellen ersten Rezeptionsphase, die entlang moralischer und ideologischer Leitlinien geführt wird, setzt enorm schnell ein Kanonisierungsprozess ein, wobei die internationale Rezeption als Katalysator wirkt: Mit Grass' Debütroman gibt es endlich wieder ein Werk, das über die nationalen Grenzen hinaus Beachtung findet und später in die

\footnotetext{
57 Heinz Ludwig Arnold, „Gespräche mit Günter Grass, in Text und Kritik 1/ıa: Günter Grass, 4-5.

$58 \quad$ Mayer (1979) 151 .

59 Ueding (1999).

6o Zitzmann (1999).
} 
Weltliteratur eingehen wird. Die Rezeption der Blechtrommel in dieser Phase ist paradigmatisch für die Literaturkritik der späten 1950er- und frühen 1960er-Jahre und bildet darüber hinaus das Meinungsklima der deutschen Nachkriegsgesellschaft und der Ära Adenauer ab. Der kleinbürgerliche Mief, in dem Grass seinen Protagonisten Oskar Matzerath aufwachsen - oder besser eben nicht aufwachsen - lässt, er ist auch in den emotionalen und empörten Kritiken noch deutlich spürbar. Auch das doktrinäre, mit dem Begriff der Entartung operierende Kunstverständnis des Nationalsozialismus scheint nicht überwunden. In den nicht wenigen Verrissen des Werkes wird mit Begriffen des Ekels und der Empörung hantiert: ,pornographisch', ,widerwärtig', ,ekelhaft', ,blasphemisch'. Die Empörung entspringt oppositioneller politischer Gesinnung und identifikatorischer Lesart. Während erstere die Rezeptionshistorie des gesamten Grass'schen Werkes begleitet, nimmt letztere ab, was zum einem dem Wandel literaturkritischer Parameter geschuldet ist und zum anderen der Historisierung des Werkes, womit eine literatursoziologische und -historische Perspektive distanziertere und analytischere Zugänge dominanter werden lässt.

Die rein inhaltlich orientierte, moralisierende und doktrinäre Bewertung bleibt auf die erste Rezeptionsphase um 196o beschränkt. Die Verfilmung durch Schlöndorff 1979 löst eine abermalige Rezeptionswelle aus, in der der Roman unbestritten als kanonisiertes Meisterwerk erscheint. Der Film wiederum ist prägend für die letzten beiden Rezeptionsphasen: Wenn von der Blechtrommel die Rede ist, schwingen die Bilder der Verfilmung häufig mit, und wie der Film reduziert sich auch die Erinnerung an den Roman auf die ersten zwei Bücher der Blechtrommel. Die Nobelpreisverleihung vergrößert den Graben zwischen Autor und Werk und zeigt auch die Divergenz der nationalen und der internationalen Rezeption, wird die Auszeichnung doch auch als Schlag für die deutschsprachige Literaturkritik gewertet. Sein Debütroman wird als Meisterwerk gefeiert, an das er niemals anschließen konnte, obwohl er selbst Die Hundejahre als seinen gelungensten Text bezeichnet und der Blechtrommel nicht die Bedeutung gibt, die ihr in der Rezeption beigemessen wird. Während die wissenschaftliche Rezeption immer neue Aspekte betont, verengt sich der Blick der Literaturkritik zunehmend auf wenige Aspekte, die in einer verknappten, schematischen Interpretation und formelhaften Zuschreibungen mündet. Im groß gefeierten Jubiläum wird deutlich, dass Die Blechtrommel nicht nur der Literatur der Nachkriegszeit ihren Stempel aufgedrück und sie in ein neues Fahrwasser gebracht hat, sondern der bedeutendste und am meisten rezipierte Roman der zweiten Jahrhunderthälfte ist. Die erzählerische Raffinesse, der groteske Humor und der Kunstgriff einer so unverwechselbaren Figur wie jener des Oskar Matzerath sind die textinhärenten Faktoren, die bis heute den Erfolg 
ausmachen. Darüber hinaus, das zeigt die Rezeption anlässlich des Jubiläums, dominiert heute eine historisch-kontextualisierende Bewertung. Es ist die dissoziierte Sicht der Generation der Nachgeborenen, die in der Blechtrommel einen adäquaten literarischen Umgang mit dem Nationalsozialismus und der Schuld der Deutschen verwirklicht sieht. Es bleibt ein Paradoxon: Grass ist der streitbarste Autor Deutschlands. Die Blechtrommel bleibt unbestritten der bis heute bedeutendste Roman.

\section{Literatur}

Anz, T., „Das Blech der frühen Jahre“, in Süddeutsche Zeitung, 06.10.1999.

Arnold, H., Text und Kritik ı/ıa: Günter Grass (München, 1988).

Baron, U., „Die späte Rettung“, in Die Welt, October 01, 1999.

Böttiger, H., „Der Sozialdemokrat als Anarch“, in Frankfurter Rundschau, October 1, 1999. Bucheli, R., „Von allen guten Geistern verlassen“, in Neue Zürcher Zeitung, April 10, 2012. Butterweck, H., „Für Günter Grass beginnt jetzt das Alter“, in Die Furche, October 7, 1999. Dotzauer, G., „Ich bin's, immer noch“, in Der Tagesspiegel, September 24, 2009, 26.

Franck, J., „Das Porträt des Deutschen als Zwerg“, in FAZ, September 19, 2009, 1-2.

Gesche, J., Aus zweierlei Perspektiven. Zur Rezeption der Danziger Trilogie von Günter Grass in Polen und Schweden in den Jahren 1958-199o (Stockholm, 2003).

Görtz, F., Günter Grass. Zur Pathogenese eines Markenbildes. Die Literaturkritik der Massenmedien 1959-1969 (Meisenheim 1978).

Görtz, F. (ed.), ,Die Blechtrommel'. Attraktion und Ärgernis. Ein Kapitel deutscher Literaturkritik (Darmstadt/Neuwied 1984).

Göttler, F., „Jetzt hört was auf, jetzt fängt was an“, in Süddeutsche Zeitung, October 1, 1999.

Greiner, U., „Verspätetes Familienfoto mit Oskar“, in FAZ, April 28, 1979.

Ignée, W., „Abschied von der Blechtrommel“, in Stuttgarter Zeitung, May 04, 1979.

Isenschmid, A., „Grass ist der Richtige“, in Tagesanzeiger, October 01, 1999.

Löffler, S., „Obszöner Gnom von gestern“, in Profil, August 20, 1979.

Loschütz, G., Von Buch zu Buch - Eine Dokumentation. (Neuwied/Berlin 1968).

Mayer, S., „Grüne Jahre für Grass: Die Rezeption in den Vereinigten Staaten“, in Text und Kritik 1/ıa: Günter Grass (München, 1979), 151-161.

Neuhaus, V., Günter Grass. Die Blechtrommel. Kommentar und Materialien (Göttingen, 2010).

N.N., „Literatur-Nobelpreis für den ,ständigen Nobelpreis-Kandidaten“', in Wiener Zeitung, October 01./02, 1999. 
Roberts, D., „,Gesinnungsästhetik'? Günter Grass, Schreiben nach Auschwitz“, in P. Lützeler (Hg.), Poetik der Autoren. Beiträge zur deutschsprachigen Gegenwartsliteratur (Frankfurt am Main, 1994), 235-261.

Schirrmacher, F., „Abschied von der Literatur der Bundesrepublik“, in FAZ, October 2, 1990.

Schmidt, K., (1999a) „Diebischer Spaß“, in Die Welt, October 1, 1999.

Schmidt, T., (1999b) „Der Preis der Vergangenheit“, in Die Welt, October 2, 1999.

Segebrecht, D., „Kein neues Bißgefühl. Zehn Jahre nach der Blechtrommel - Günter Grass' dritter Roman Örtlich betäubt", in Frankfurter Allgemeine Zeitung, August 16, 1969.

Steinfeld, T., „Präzeptor“, in Frankfurter Allgemeine Zeitung, October 02, 1999.

Thomas, C., „Einfühlung und Horrorwörter. Die Tabubruchkonkurrenzen bei Günter Grass", in Frankfurter Rundschau, April 16, 2012, 21.

Thuswaldner, A., „Böser Bube, guter Dichter“, in Salzburger Nachrichten, October 1, 1999.

Ueding G., „Die Sprache des Nobelpreisträgers“, in Die Welt, October 2, 1999.

vw [Kürzel nicht aufgelöst], „Das Warten hat ein Ende: Nobelpreis für Günter Grass“, in taz, October 10, 1999, 1.

Weidermann, V., „Es ist ein ekelhaftes Gedicht“, in FAZ, April 8, 2012.

Wittstock, U., „Drei Meilensteine, eine Zäsur“, in Die Welt, March 12, 2009, 23.

Wieckhorst, V., „Der Blechtrommler“, in Rheinischer Merkur, October 8, 1999.

Zitzmann, M., „Une grande gueule“, in Neue Zürcher Zeitung, October 13, 1999. 